\title{
Correction to: Methods to Detect Immunogenic Cell Death In Vivo
}

Takahiro Yamazaki, Aitziber Buqué, Marissa Rybstein, Jonathan Chen,
Ai Sato, and Lorenzo Galluzzi

\section{Correction to:}

Chapter 20 in: Magdalena Thurin et al. (eds.), Biomarkers for Immunotherapy of Cancer: Methods and Protocols, Methods in Molecular Biology, vol. 2055, https://doi.org/10.1007/978-1-4939-9773-2_20

This book was inadvertently published with one of the contributing author's name printed as Aitziber Buqué Martinez and it should have been Aitziber Buqué. This correction has been updated in the book.

The original version of this chapter was revised. The updated version of this chapter is available at https://doi.org/10.1007/978-1-4939-9773-2_20 